\title{
LA VALEUR APPORTÉE PAR UN RÉSEAU SOCIAL BRICK AND MORTAR : LES POINTS PASSERELLE DU CRÉDIT AGRICOLE
}

Les banques mutualistes à force de vouloir grandir ont souvent perdu de vue les valeurs qui ont prévalues à leur fondation. Le Crédit Agricole, et tout particulièrement sa caisse Nord Est, a développé un réseau social, qui loin d'être virtuel, crée une solidarité concrète entre de nombreuses parties prenantes. L'auteur analyse dans le détail les performances économiques et sociales de ce réseau en utilisant la logique de « service dominant », et pose la question de l'évolution de ce type de structure en période de crise.

\section{Par Anne JULIEN*}

Nous vivons à une époque où tout échange est assorti d'un service. C'est la « logique de service dominant » avec ses différents corollaires : une coproduction accrue entre le fournisseur et le client, la construction de multiple réseaux de service et la mesure de ces réseaux pour favoriser la co-création de la valeur par les différentes parties prenantes.

Peu de travaux réalisés dans le domaine bancaire ont eu recours à cette approche. D'autres causes plus contextuelles nous ont également poussée à orienter nos travaux dans ce secteur. Suite aux différents soubresauts que connaît le secteur financier, un certain nombre de banques sont en train d'opérer un recentrage sur le développement de leur territoire. Elles communiquent et cherchent à agir dans ce sens. Dans le même temps, les ménages vulnérables ont à faire face à des difficultés exacerbées par la crise. II serait donc intéressant de trouver des pistes susceptibles de favoriser le rôle économique et social que l'on attend des établissements de crédits, et plus spécifiquement des établissements régionaux et mutualistes.

Dans cet article, nous étudierons le réseau des «Points Passerelle » du Crédit Agricole, qui co-roduisent avec des habitants en difficulté et avec d'autres acteurs des secteurs privé et public.

Par le biais des méthodes issues de la logique de service (VARGO et LUSH, 2008), nous chercherons à savoir pour quelles raisons, de quelle manière et dans quels buts, les différents acteurs interagissent entre eux. Cette analyse doit nous permettre de répondre aux questions suivantes : comment les acteurs créent-ils de la valeur? Comment ce système peut-il être viable ? Jusqu'où une banque coopérative peut-elle appliquer ses valeurs mutualistes ? Pour finir, nous développerons des recommandations pour permettre l'adaptation de ce réseau, qui comprend de multiples ramifications et est soumis à de fortes contraintes tant économiques qu'organisationnelles et sociales.

\section{CADRE THÉORIQUE}

Le recours aux concepts de coproduction, co-création et de logique de service dominant 
La logique de service dominant (SDL, ou Service Dominant Logic) repose sur la constatation, qu'au $\mathrm{XXI}^{\mathrm{e}}$ siècle, dans tout échange, il y a une part de service, et que le résultat du service proposé par le fournisseur dépendra plus ou moins largement du rôle joué par les clients.

En effet, ces derniers jouent un rôle actif dans la création de services et le développement de leurs bénéfices personnels (PRAHALAD et RAMASWAMY, 2003). Le concept de la participation de la clientèle existe depuis de nombreuses années (EIGLIER et LANGEARD, 1987). La nouveauté réside dans le fait que les prestataires apportent de moins en moins d'éléments dans le processus de création de valeur par les clients. Ils n'en sont que les initiateurs. Le rôle de coproducteur des clients devient alors de plus en plus prégnant et revêt un caractère quasi obligé. (GRÖNROOS, 2008 ; GUMMESSON, 2007). Ce comportement est renforcé par l'éducation supposée croissante des clients, la multiplicité des offres proposées en libre-service et le souhait d'autonomie exprimé par de nombre de consommateurs. Toutefois, certaines personnes ne possèdent pas les capacités ou les attitudes pour développer cette autonomie, et leurs connaissances dans certains domaines, comme celui de la finance, restent faibles.

\section{Les réseaux de services}

La coproduction se traduit par une valeur évaluée par le client comme l'écart entre les différents coûts constatés et les différents bénéfices tirés de cet échange. Certains auteurs (NGO et O'CASS, 2009) ont cherché à comprendre comment l'offre de valeur était intégrée à plusieurs niveaux. Ils ont notamment examiné le rôle joué par des ressources issues de l'échange de compétences spécialisées ou de connaissances, plutôt que de s'intéresser à des ressources matérielles. Ces échanges sont favorisés par une proposition de valeur créée en réseau, voire en tribu, plutôt qu'avec un seul interlocuteur (CovA et SALLE, 2008).

Les différents acteurs ont un rôle d'intégrateur de ressources, « où les acteurs économiques et sociaux interfèrent au sein de réseaux qui interagissent avec d'autres réseaux 》 (VARGO et LuSH, 2008).

\section{Les mesures des réseaux de services}

Ces différents réseaux constituent un système de services qu'il est nécessaire d'évaluer.

Les quatre principaux axes de mesure sont:

- la qualité ;

- la performance ;

- le respect ;

- et l'innovation durable.

À partir de ces mesures et de l'approche dite «Approche de la viabilité du système (VSA) 》, le système qui perdure sera qualifié de réseau de services intelligents ou viables (BARILE et POlese, 2010). 
Pour obtenir une analyse plus fine, la réflexion peut être poussée sur la base des dix éléments suivants : les ressources, les entités, les droits d'accès, la co-création de valeur résultant des interactions, la gouvernance, les résultats, les intervenants, les mesures, les réseaux et l'écologie.

Impulsées par la gouvernance, les interactions doivent créer l'efficacité, l'efficience et la viabilité. De plus, les résultats produits doivent satisfaire les différents types d'intervenants que sont les parties prenantes (MELE et al., 2010).

Les études basées sur les réseaux de services sont largement issues du secteur de l'informatique ou de celui de la technologie. L'étude empirique que nous avons réalisée porte sur un système élaboré en France, dans le secteur financier. Nous allons maintenant nous intéresser à l'environnement dans lequel s'exercent ces activités.

\section{Les réseaux de services dans le secteur financier}

En France, mais aussi dans de nombreux autres pays, la majorité des banques considèrent leurs actionnaires comme leur principale partie prenante. C'est pour cette raison que pendant des années, les banques se sont attachées à développer leur chiffre d'affaires et leur rentabilité en poussant de nombreux produits vers leurs clients. Aujourd'hui, les établissements ont pour objectif majeur de satisfaire leurs clients et de créer une fidélité active, tout en diminuant leurs risques.

Se référant aux propos de Gummesson (2008) qui affirme que "le service est créé dans un réseau d'activités impliquant une multitude d'intervenants ", les chercheurs comme les praticiens ont développé le concept de balanced centricity, en vertu duquel chaque partie prenante a le droit d'être satisfaite.

Cette voie se rapproche du courant mutualiste, tel qu'il a été pensé à ses origines. Au fil des années, ces banques coopératives ont souvent perdu les valeurs de solidarité et d'égalité qui les animaient lors de leur création au profit d'une rentabilité financière qui leur a permis de se développer. Toutefois, les banques coopératives régionales souhaitent aujourd'hui revenir à leurs valeurs originelles, qui prônaient la proximité et le partage avec les clients. En ce sens, elles cherchent à promouvoir leur responsabilité sociale et sociétale à partir de services tangibles. Elles parlent d'opérations organisées au bénéfice de tous, y compris des plus démunis. Mais qu'en est-il concrètement sur le terrain ?

\section{CADRE EMPIRIQUE : L'ÉTUDE DE CAS}

Nous allons maintenant décrire le réseau des Points Passerelle à partir d'observations et d'entretiens que nous avons rassemblés lors de notre étude qui s'est inscrite dans la durée (trois ans), ce qui nous a permis de suivre l'évolution de la structure et de ses acteurs.

\section{La structure du réseau social}

\section{Historique et raison d'être du service Passerelle}

Le service Passerelle a été créé en 1997 au sein du Crédit Agricole Nord Est. Cette structure a évolué sous la forme d'une association, en 2001. La Caisse Régionale de Crédit 
Agricole Mutuel du Nord Est, dont le siège est situé à Reims, couvre trois départements (la Marne, l'Aisne et les Ardennes). L'idée de départ était de créer des passerelles, au travers de conseils et un accompagnement humain, avec des personnes traversant une grave crise dans leur vie. Le but premier était de leur permettre d'échapper à un triple danger : celui de l'exclusion économique (perte de revenus, destruction du patrimoine de précaution), celui de l'exclusion bancaire (disparition des principaux moyens de paiement indispensables à la vie courante) et, enfin, le risque de l'exclusion sociale (perte du lien d'appartenance à la famille, au groupe social, au voisinage, à la cité,...).

Un point d'accueil Passerelle a été installé dans cinq villes principales : Reims, Chalons-enChampagne, Charleville, Laon et Château-Thierry. Les locaux sont situés en dehors des agences bancaires et sont identifiés par une signalétique discrète : "Point-Passerelle Nord Est ». Les Points Passerelle (PP) sont des espaces d'écoute qui s'inscrivent dans le cadre d'un savoir-faire bancaire et financier, avec des salariés rémunérés par la banque qui reçoivent, orientent et accompagnent les clients, lesquels sont appelés les requérants. Pour bénéficier de ce soutien, les personnes concernées doivent avoir exprimé la volonté de se prendre en charge afin de faire face à leurs difficultés, c'est la condition essentielle pour que le dispositif fonctionne, c'est-à-dire pour que la coproduction de valeur soit effective. Ces personnes ne sont pas nécessairement clientes du Crédit Agricole.

\section{Les acteurs}

Dans chaque Point Passerelle, deux animateurs salariés accueillent et conseillent, au quotidien, les personnes en difficulté. Un directeur est responsable du bon fonctionnement de l'ensemble des Points. II instruit les dossiers qui doivent être examinés en commission. II établit des relations avec les différents partenaires et anime le réseau des accompagnateurs.

Les animateurs des Points Passerelle sont donc des salariés du Crédit agricole et sont volontaires pour exercer ces fonctions. Leur sélection s'opère sur trois critères : leur motivation pour le poste, leurs valeurs humanistes et un solide équilibre psychique. Ils sont placés en disponibilité auprès du service Passerelle pendant quelques années, et sont appelés à reprendre ultérieurement une fonction bancaire classique.

Leur mission est d'accueillir les personnes en difficulté, c'est-à-dire, dans un premier temps, de les écouter de manière bienveillante, en faisant preuve d'humanité, sans a priori. $\mathrm{Ce}$ moment est essentiel, parce qu'il leur permet d'aider le demandeur à identifier son problème, à en clarifier les composantes, à le resituer dans le contexte global de sa vie, et à rechercher des solutions. Après une phase d'écoute et de diagnostic, les conseillers ont pour mission de rechercher, en partenariat avec l'ensemble des acteurs sociaux, des solutions permettant de remettre en selle, voire de « resolvabiliser » les personnes aidées. Souvent, les " sinistrés du crédit » ne sont surendettés qu'en apparence : si les mensualités sont certes insurmontables, le capital emprunté est par contre modéré. Ils répondent aux critères du «mal endettement ». Si les personnes viennent avant tout pour des problèmes financiers, il arrive aussi qu'elles aient besoin de parler de problèmes plus personnels.

L'action de ces animateurs est prolongée par celle, indispensable, des accompagnants bénévoles. Un réseau de cent vingt accompagnateurs a été constitué pour assurer le suivi et l'aide personnalisée. Il est composé de retraités bénévoles du Crédit Agricole du Nord Est, d'administrateurs des caisses locales ou encore de personnes 
motivées ayant suivi au minimum une formation à l'accompagnement. L'accompagnant rencontre régulièrement l'emprunteur confronté à des difficultés de remboursement.

\section{Les requérants}

Ils viennent soit en tant que clients des agences du Crédit Agricole, soit sur les conseils de prescripteurs externes. Il faut noter que les animateurs des Points Passerelle ont l'habitude de travailler avec certains conseillers de la banque qui proposent l'aide de ces points à leurs clients confrontés à des difficultés financières, pour éviter à ces derniers de relever de la procédure de surendettement de la Banque de France.

Les situations les plus fréquemment rencontrées sont celles liées à un chômage brutal, alors que le crédit contracté pour acheter le logement n'est pas encore définitivement remboursé. Les difficultés liées au chômage s'accompagnent souvent d'autres facteurs d'exclusion, comme le divorce. Les profils des dossiers étudiés correspondent le plus souvent à des familles monoparentales, des femmes seules avec des enfants. II s'agit le plus souvent d'une situation d'endettement passif.

Il existe une frange de la population, se situant à la limite de l'exclusion, qui peut s'en sortir si et seulement si d'autres personnes leur viennent en aide en leur prodiguant les bons conseils, notamment au niveau des procédures administratives et de la gestion du budget, et, bien sûr, s'ils sont prêts à s'impliquer.

Ce sont le plus souvent des jeunes, mais on voit venir aujourd'hui des retraités qui n'avaient pas bien intégré la baisse de leurs revenus. Cet état de fait est aggravé par la crise économique, et s'observe plus spécifiquement dans certaines zones urbaines ou semiurbaines où les liens sociaux se sont délités, contrairement aux « zones rurales à l'ancienne » où existe encore une certaine solidarité (les Ardennes nord, la Thiérache).

\section{Les évolutions du système}

\section{La Caisse Locale de Développement Partagé}

Les animateurs des Points Passerelle se sont rapidement aperçus que l'accès au crédit était impossible pour les requérants, alors qu'il pouvait être un outil précieux pour des cas très spécifiques. À partir de 1998, ces Points ont expérimenté quinze crédits solidaires totalement hors normes, sur décision de la direction générale ; le succès a été total...

Dès lors, quelle structure doit-on mettre en place pour pérenniser ce type de crédit?

Ainsi, pour compléter le dispositif, la Caisse Régionale a créé, en 2001, une Caisse Locale de Développement Partagé (CLDP). Celle-ci est l'instrument bancaire spécifique de la mise en œuvre de la politique solidaire de la Caisse. Elle apporte une solution spécifiquement bancaire à des personnes en difficulté : crédit solidaire pour financer le lancement de projets d'activités économiques portés par des chômeurs, prêts dits de « resolvabilisation » octroyés à des personnes en situation d'insolvabilité, crédits attribués de manière préventive pour prévenir une situation intenable à court terme, ou pour restaurer une situation financière et éviter ainsi aux personnes concernées de relever de la Commission de surendettement, ou encore, se substituer à cette dernière lorsque la personne n'est pas éligible à cette Commission. 
La CLDP applique le principe de subsidiarité : elle réalise les opérations que les soixantedix neuf autres Caisses Locales de la banque ne sont pas habilitées à traiter en vertu d'une réglementation interne de la banque régionale. Un comité chargé du traitement des dossiers se réunit tous les 15 jours. Il examine donc les dossiers, en analysant notamment les circonstances qui ont conduit les requérants à se retrouver en situation de difficulté, et vérifie que ceux-ci aient la volonté et les capacités de gérer au mieux leur budget.

Les emprunteurs vont faire l'objet systématiquement d'un accompagnement personnalisé pendant toute la période de leur «convalescence » économique.

Les prêts solidaires présentent obligatoirement les spécificités suivantes :

- le raisonnement adopté pour apprécier l'opportunité du prêt tient compte du « reste à vivre » et non pas de la détermination d'une capacité d'emprunt par rapport aux revenus disponibles ;

- un accompagnement personnalisé ;

- un suivi du dossier qui repose sur une concertation entre le service Passerelle et les accompagnateurs et l'agence bancaire ;

- et la durée, qui court jusqu'à la restauration complète de l'autonomie et de la maîtrise budgétaire.

La Caisse Locale de Développement Partagé est une véritable banque solidaire, mettant tous ses outils de crédit à la disposition des acteurs en lutte contre la fragilisation et l'exclusion.

Au final, près de deux personnes sur dix franchissant le seuil d'un Point Passerelle vont bénéficier d'un emprunt solidaire (17\% plus exactement).

Il existe également un comité solidaire qui traite de quelques dossiers par an : ce sont des personnes qui ont besoin d'un suivi ou d'une médiation bancaire et sociale, qui exigent la participation de la direction générale du Crédit Agricole. Ce comité est en charge des cas les plus difficiles, ceux identifiés et présentés par le directeur des Points Passerelle. Mais outre cette transmission, ces cas peuvent être présentés par l'intermédiaire des Points Passerelle, du service client ou du service contentieux de la banque. Ils pourront faire l'objet, par exemple, d'un abandon partiel ou total de créances.

\section{Les contraintes juridiques et réglementaires}

Les Points Passerelle ne peuvent pas être des agences bancaires proprement dites. L'activité de crédit solidaire est en fait directement prise en charge par la banque et ses conseillers spécialisés.

\section{Le conseiller solidaire}

Pour pallier ces contraintes juridiques et rendre le processus complètement transparent, la banque régionale a créé trois postes de conseiller solidaire, un par département. Ces derniers vont s'occuper plus spécifiquement de l'instruction du crédit en liaison avec la CLDP et des relations avec l'agence bancaire.

\section{Les contraintes fiscales}

Les activités décrites ci-dessus représentent une charge pour la banque qui a pris la décision de les faire financer pour partie par sa propre fondation, plutôt que par une 
dotation de la banque à une association. Les fondations d'entreprise permettent en effet de bénéficier d'avantages fiscaux non négligeables. En contrepartie, les activités doivent répondre à des objectifs précis et définis dans le temps ; ces activités doivent être contrôlées par un auditeur externe. Le conseil d'administration de la fondation comprend des membres de l'entreprise et des personnes extérieures connaissant bien le secteur d'activité considéré.

Au final, l'organisation du réseau des Points Passerelle présente en 2013 une séparation nette entre les activités strictement bancaires et tout ce qui se rapporte à la gestion des relations avec d'autres types de réseaux qui interviennent aussi dans la vie des requérants.

Insérer Figure 1 : Organisation du réseau de services : Les Points Passerelle

\section{ANALYSE ET DISCUSSION}

Ce schéma qu'il faut inclure dans un environnement économique, social et environnemental plus large, nous montre la complexité de ce système dès lors qu'il comprend plusieurs réseaux (NORMANN et RAMIREZ, 1993). Les entités du système des Points Passerelle interagissent avec d'autres entités : par exemple, le réseau des partenaires interagit avec les animateurs des $\mathrm{PP}$, avec les requérants, avec les agences bancaires... La co-création générale se fait avec de nombreux acteurs et mobilise de multiples ressources.

\section{Les différents critères de mesure des réseaux}

Nous allons procéder à une première analyse sur la base du tableau suivant, qui reprend les dix points de la mesure des réseaux que nous avons évoqués au début de notre article.

\section{Insérer Tableau 1 : les différentes mesures}

Analysons maintenant les résultats de manière plus transverse afin de nous assurer que les objectifs d'un réseau intelligent ou viable (qualité, performance, respect, innovation durable) sont bien présents.

De quelle viabilité est-il question ? Quels sont les résultats attendus ?

\section{Premier résultat : la qualité}

Les personnes faisant appel au réseau Passerelle pour tenter de « se remettre à flot » sont avant tout des acteurs économiques. "Une plaie d'argent n'est pas mortelle, une plaie d'argent peut se soigner, et Passerelle peut vous y aider », nous dit Madame V. (une requérante). Ces personnes travaillent, elles produisent, consomment, épargnent, investissent dans la région et contribuent ainsi à sa bonne santé économique. S'ils tombent dans l'exclusion, ils appauvrissent de fait le tissu économique global. Le rétablissement de la solvabilité de ces clients grâce à l'aide du réseau PP est synonyme d'une consommation potentielle que l'on ne peut pas négliger. Cela est illustré par les résultats du tableau 1. Les subventions allouées à Passerelle servent à aider des personnes à redevenir des clients comme les autres. Le montant de cette aide représente de fait une part non négligeable des bénéfices de cette banque. 
Insérer Tableau 2 : Les résultats des actions menées dans la Caisse du C.A. Nord Est

\section{Deuxième résultat positif : la performance économique et sociale}

Chaque année, 5000 entretiens sont réalisés, et 1000 à 1200 nouveaux contrats sont signés. Depuis la création des Points Passerelle, près de 11000 personnes ont été accueillies (dont près de $17 \%$ ne sont pas des clientes du Crédit Agricole du Nord Est). Les problèmes rencontrés sont d'ordre financier dans $70 \%$ des cas.

Il y a 600 dossiers de crédit en encours d'un montant moyen de 17750 euros.

Les risques que présentent ces prêts sont de même niveau que ceux encourus avec des professionnels, cela tient au fait que les bénéficiaires de ces prêts solidaires sont accompagnés et même soutenus par de nombreux coproducteurs : les salariés de la banque et les accompagnateurs (GIANFALDONI et RICHEZ-BATTESTTI, 2006). Cet accompagnement multiplie par cinq ou six les chances de remboursement de l'emprunt, ce qui fait que le taux de défaut pour les crédits solidaires est très proche du taux de défaut moyen constaté en France, soit $4 \%$.

\section{Troisième résultat : Le respect}

Les personnes en charge des requérants disent qu'ils agissent comme des conseillers financiers, à la différence qu'ils ne sont pas soumis à des objectifs commerciaux mais à de forts objectifs touchant à l'aspect humain. « Le Point Passerelle par la mise en place d'un prêt solidaire adapté à mes possibilités de remboursement m'a permis de sortir d'une impasse », précise Madame D. (une requérante). "Le Point Passerelle est un lieu où l'on prend le temps de vous écouter, où l'on est considéré », ajoute Monsieur M. (un autre requérant).

Les accompagnants retraités du secteur bancaire connaissent leur métier. Ils comprennent les difficultés des requérants. II n'y a pas de jugement, seulement une volonté partagée, celle que le requérant s'en sorte.

De plus, des conventions ont été signées avec des partenaires comme EDF (le service Passerelle est référencé comme partenaire social d'EDF) ou des bailleurs sociaux qui peuvent ainsi contribuer à alléger les charges des requérants.

\section{Les derniers résultats mis en évidence se rapportent à l'innovation durable}

Cette notion pourrait être associée à celle de responsabilité sociale des entreprises (SCHOLTEN 2006 ; WEAVER et al., 1999) : “En comparaison avec les caractéristiques spécifiques du Crédit Agricole qui est une banque mutualiste, l'association Point Passerelle donne un sens aux résultats de notre Caisse régionale. En effet, la Passerelle fournit des conseils, une assistance et souvent des solutions - c'est-à-dire une vision nouvelle et différente des personnes vulnérables et des victimes de la vie ", nous dit un sociétaire impliqué dans le réseau.

"Nos directeurs demeurent les acteurs locaux de leur conseil d'administration, et des ambassadeurs actifs du système de solidarité dans leur région. Certains acceptent de 
suivre nos requérants à titre d'entraîneurs, de jouer un rôle actif dans le suivi du budget et de contribuer à la bonne marche de nos affaires ", déclare le nouveau directeur des Points Passerelle.

"L'accompagnateur sera le soutien, le confident de la personne ; il devra faire preuve de psychologie pour se faire accepter et de pédagogie pour aider la personne à apprendre ou réapprendre à gérer son budget familial », nous précise Monsieur X (un accompagnateur Passerelle).

Si une banque mutualiste cultive les valeurs de la solidarité, elle se doit dès lors d'aider ses clients dans les moments difficiles et pas seulement être présente dans les bons moments. D'une part, cela correspond à une performance sociale des entreprises et, d'autre part, cela permet une réduction des coûts opérationnels associés à la gestion des litiges générés par une prise en charge trop tardive des problèmes. Par conséquent, le système se traduit par une diminution des risques globaux de l'entreprise en prenant des risques contrôlés par le jeu d'un accompagnement des requérants (GRANOVETTER, 2005).Toutefois, il convient de noter, depuis 2011, un développement significatif des dossiers (plus d'un tiers) arrivant au PP qui ne peuvent qu'être transférés à la Banque de France.

Au final, les objectifs visant à poursuivre le développement du mouvement mutualiste et à rendre son action porteuse de sens ont été respectés.

On constate donc qu'aujourd'hui, ce système fonctionne de façon viable et efficace, avec des chiffres de requérants qui restent stables.

\section{Quel avenir pour ce type de réseaux de services?}

Quel est l'avenir de ce système au niveau de l'ensemble des caisses régionales du Crédit Agricole?

En 2007, le congrès de la Fédération du Crédit Agricole - l'instance politique de la banque - a demandé aux caisses de développer ces structures. Fin 2008, 20 caisses, soit plus de la moitié des caisses régionales, ont mis en œuvre le dispositif. 33 Points Passerelle étaient opérationnels dans la France entière, dont un Point Escale en Bretagne.

Fin 2011, on dénombrait 66 Points ouverts pour 29 caisses régionales. Ces Points Passerelle ont adopté soit le modèle complet comme celui développé par la caisse Nord Est, soit le seul volet écoute solidaire. Seuls cinq modèles associatifs existent à l'échelle de la France. Au total, 120 conseillers sont détachés auprès du service Passerelle, et on dénombre près de 600 accompagnants bénévoles. Ainsi, ce sont plus de 9000 requérants qui ont bénéficié d'un soutien en 2011 à l'échelle du territoire national, avec une forte augmentation des dossiers au titre des années 2010 et 2011.

Que ce soit pour le Crédit Agricole ou pour d'autres banques, les principales contraintes de ce système de services centré sur le réseau des Points Passerelle sont les suivantes:

- Les risques liés aux différents réseaux

S'assurer de la détermination des requérants à se prendre en main (ZWICK et al., 2008). « Je recommanderai le système sous réserve que la personne veuille [réellement] s'en 
sortir. Il faut accepter les contraintes, faire des efforts, mais cela en vaut la peine. Aujourd'hui, je vis plus sereinement », nous fait part Monsieur M. (un requérant). Cette façon de sélectionner les requérants est aussi une façon de réduire les risques ;

- la nécessité absolue d'accompagner l'emprunteur dans la gestion de ses crédits de façon à diminuer le taux des défauts de paiement. II faut aussi veiller à renouveler le vivier des accompagnateurs.

- la crainte des dirigeants de la banque de voir le nombre des personnes accueillies dans les Points Passerelle fortement augmenter est toujours présente, même si dans les faits elle n'est pas vérifiée, puisque la population des requérants reste stable alors même que la population des personnes surendettées ou considérées comme pauvres ne cesse de croître ;

- la nécessité de mieux informer et de sensibiliser l'ensemble des conseillers de clientèle du réseau traditionnel : les animateurs des Points Passerelle ont en effet pris l'habitude de travailler avec un nombre restreint de conseillers de la banque, ce qui limite les opportunités pour les clients fragiles de pouvoir s'en sortir.

\section{- Le financement du réseau des Points Passerelle}

Quel est le budget alloué à ces actions par les banques ? Comment ce budget va-t-il évoluer ? Comment bien mesurer la rentabilité de ces actions pour la banque?

Ce modèle de financement doit-il être uniquement basé sur des entreprises privées, ou doitil s'ouvrir à des partenariats avec des organismes publics ou d'autres associations ? Une évolution qui obligerait à adopter une gouvernance élargie.

- Le management à l'intérieur du réseau des Points Passerelle.

La difficulté à manager un tel système tient au fait qu'il repose avant tout sur un fort investissement humain pour pouvoir gérer des cas à chaque fois différents : "On ne peut pas industrialiser notre intervention, dans certains cas cela s'apparente à du coaching, à du conseil dans l'accompagnement, parfois à de la médiation dans les couples, parfois encore à de la négociation avec les banquiers », nous précise Monsieur G. (un animateur d'un point Passerelle).

\section{- La communication vis-à-vis des différentes parties prenantes}

Il est toujours difficile d'adopter une communication éthique sur ce type d'actions. Toutefois, le fait de passer par une fondation permet d'assurer une plus grande transparence dans la diffusion de l'information ;

Comment communiquer en dehors des assemblées générales sur des actions qui véhiculent des valeurs d'éthique de la banque ? Des efforts ont été faits récemment pour mieux informer les sociétaires et les clients (au travers de discours et la diffusion de dépliants) ;

Comment répondre aux attentes de consommateurs qui évaluent de plus en plus les marques sur leurs comportements, tout particulièrement dans le secteur bancaire, sans pour autant que les actions des Points Passerelle apparaissent comme un système de rattrapage ou un simple alibi? 


\section{CONCLUSION}

Pour permettre à des personnes fragilisées de se « remettre en selle », le réseau des Points Passerelle, interconnecté avec d'autres réseaux sociaux, représente une proposition de valeur qu'apprécient les habitants de la région. II se présente sous la forme d'un réseau de services qui apporte aux personnes considérées un soutien, une écoute active et la possibilité d'avoir accès à l'emprunt solidaire. Cette étude apporte un éclairage complémentaire à la logique de service dominant qui concerne à la fois les services privés et les services publics. La viabilité de ce système qui s'exprime au travers d'un nombre de requérants quasiment stable est donc manifeste, mais elle pose un certain nombre de questions.

S'agissant des prêts, les plans de " solvabilité restauration » pourraient être mis en œuvre, sur le plan technique, par d'autres banques nationales ou régionales dans le but de lutter contre "le crédit inapproprié ou le mal endettement ", sous la réserve expresse qu'il y ait un accompagnement des emprunteurs. Ces micro-crédits peuvent être considérés comme des outils de (re)conquête de la clientèle, à l'image du Crédit Agricole, qui, à ses origines, s'occupait des agriculteurs alors que les autres banquiers ne souhaitaient pas le faire.

Toutefois, un accroissement de cette co-production solidaire nécessiterait de mettre en place une stratégie différente. II pourrait s'agir d'innover, seul ou en partenariat avec d'autres. Le Crédit Agricole et ses filiales d'assurances sont engagés dans une réflexion (déjà bien avancée) sur comment créer un véritable business social en proposant de nouveaux produits et services adaptés à ces personnes en situation de fragilité.

Le système de réseaux analysé ici permet à la banque de recréer une dynamique économique et sociale qui doit favoriser à terme ses activités bancaires. Cependant, un tel système se situe à la croisée des chemins. Face à la montée de la paupérisation, il se maintient aux côtés d'autres acteurs grâce à la politique de la banque régionale. Faut-il que celle-ci adopte une nouvelle stratégie visant à accroître sa visibilité, ce qui permettrait de toucher une frange plus large de la population susceptible de bénéficier du soutien des Points Passerelle, et ce, bien avant que cette population soit concernée par la procédure d 'inscription sur le fichier des interdits bancaires de la Banque de France ? Va-t-elle continuer à assurer ce type financement qui est essentiellement mis en avant lors des assemblées générales de sociétaires ? Jusqu'où un tel investissement est-il supportable dans un secteur où les business models sont en train de fortement évoluer? 
NOTE

* Enseignant chercheur, Reims Management School, Titulaire de la Chaire Bancassurance Crédit Agricole du Nord Est, Membre du laboratoire de Recherche Magellan (Lyon 3) 


\section{BIBLIOGRAPHIE}

BARILE (S.) \& POLESE (F), "Linking the viable system and many-to-many network approaches to service-dominant logic and service science", International Journal of Quality and Service Sciences, vol. 2, n¹, pp. 23-42, 2010.

COVA (B.) \& SALLE (R.), "The Industrial/consumer marketing dichotomy revisited", Journal of Business and Industrial marketing, vol. 23, n¹, pp. 3-11, 2008.

EIGLIER (P.) \& LANGEARD (E.), «Servuction : le marketing des services », Paris, MCGraw-Hill, Ediscience international, 1987.

GIANFALDONI (P.) \& RICHEZ-BATTESTI (N.), Gouvernance et proximité : la contribution des banques coopératives au développement Local, article présenté lors des Cinquièmes Journées de la Proximité «La proximité, entre interactions et institutions », Université de Bordeaux, pp. 4-28, 2006.

GRANOVETTER (M.), "The Impact of Social Structure on Economic Outcomes", Journal of Economic Perspectives, vol. 19, n¹, pp. 33-50, 2005.

GRÖNROOS (C.), "Service logic revisited: who creates value? Ad who co-creates?", European Business Review, vol. 20, n², pp. 298-314, 2008.

GUMMESSON (E.), "Exit Services Marketing - Enter Service Marketing", The Journal of Customer Behaviour, vol. 6, n², pp. 113-141, 2007.

GUMMESSON (E.), "Extending the New Dominant Logic: From Customer Centricity to Balanced Centricity", Commentary for Special Issue of the Journal of the Academy of Marketing Science on Service Dominant Logic, vol. 36, n¹, pp. 15-17, 2008.

MELE (C.), PELS (J.) \& POLESE (F.), "A Brief Review of Systems Theories and Their Managerial Applications", Service Science, vol. 2, n¹/2, Spring-Summer, pp. 126-135, 2010.

NGO (L.) \& O'CASS (A.G.), "Creating value offering via operant resource-based capabilities", Industrial Marketing Management, vol. 38, n¹, pp. 45-59, 2009.

NORMANN (R.) \& RAMIREZ (R.), "From value chain to value constellation : designing interactive strategy", Havard Business Review, vol. 71, n7/8, pp. 65-77, 1993.

PRAHALAD (C.K.) \& RAMASWAMY, (V.), "The New Frontier of Experience Innovation", Sloan Management Review, vol. 44, n4, pp. 12-18, 2003.

SCHOLTEN (B.), "Finance as a driver of Corporate Social Responsibility", Journal of Business Ethics, vol. 68, n¹, pp. 19-33, 2006.

VARGO (S.L.) \& LUSCH (R.F.), "Service-Dominant Logic: Continuing the Evolution", Journal of the Academy of Marketing Science, vol. 36, pp. 1-10, 2008.

WEAVER (G.), TREVINO (L.) \& COCHRAN (P.), "Integrated and Decoupled Corporate Social Performance: Management Commitments, External Pressures and Corporate Ethics Practices, Academy of management Journal, vol. 42, n5, pp. 539-552, 1999.

ZWICK (D.), BONSU (S.K.) \& DARMODY (A.), "Putting consumers to work: 'co-creation' and new marketing govern-mentality", Journal of Consumer Culture, vol. 8, n², pp. 163196, 2008. 
Figure 1 : Organisation du réseau de services : Les Points Passerelle

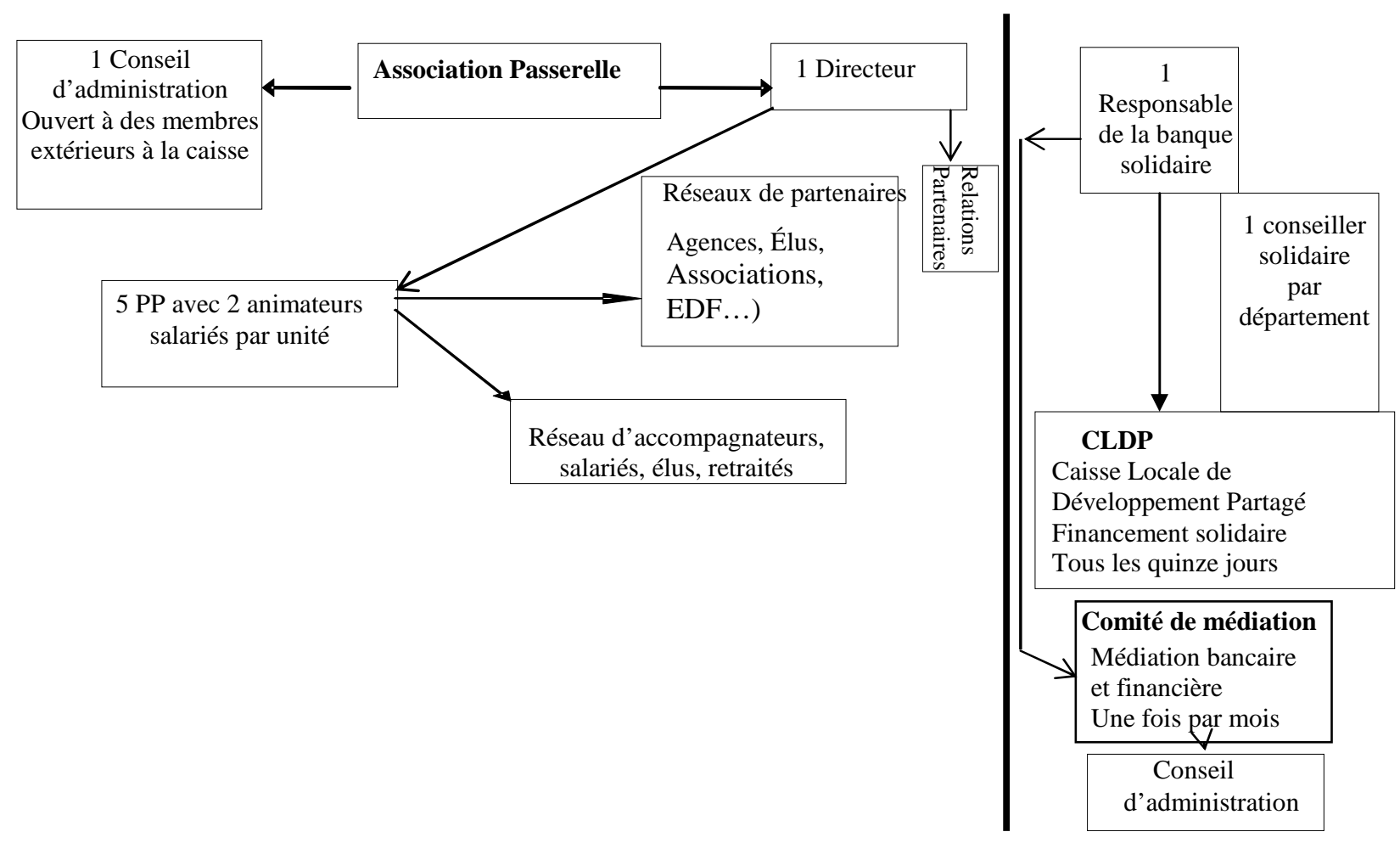


Tableau 1 : les différentes mesures

\begin{tabular}{|c|c|}
\hline Les ressources & $\begin{array}{l}\text { Une partie des ressources de l'association } \\
\text { vient de la fondation. L'autre partie vient } \\
\text { directement de la banque Crédit Agricole } \\
\text { du Nord Est. }\end{array}$ \\
\hline Les entités & $\begin{array}{l}\text { Associations, CDLP, comité de la banque, } \\
\text { entreprises partenaires publiques ou } \\
\text { privées. }\end{array}$ \\
\hline Les intervenants & $\begin{array}{l}\text { Salariés, accompagnateurs et conseillers } \\
\text { solidaires responsables de la banque, } \\
\text { accompagnateurs bénévoles, sociétaires. }\end{array}$ \\
\hline Les droits d'accès & $\begin{array}{l}\text { Pas de droit d'accès (comme celui d'être } \\
\text { client de la banque) mais une obligation de } \\
\text { vouloir s'en sortir. }\end{array}$ \\
\hline La co-création de valeur des interactions & $\begin{array}{l}\text { Partage des connaissances budgétaires et } \\
\text { administratives, écoute attentive, aide à } \\
\text { l'inclusion bancaire et sociale. }\end{array}$ \\
\hline La gouvernance & $\begin{array}{l}\text { Conseil d'administration de la Fondation, } \\
\text { de la CLDP, de l'association Passerelle } \\
\text { (dont les administrateurs sont des } \\
\text { membres extérieurs à la banque, ce qui } \\
\text { veut dire que les administrateurs ne sont } \\
\text { pas les payeurs) et le directeur des Points } \\
\text { Passerelle. }\end{array}$ \\
\hline Les résultats & $\begin{array}{l}66 \% \text { de succès dans la réinsertion (qui } \\
\text { peut être totale ou partielle). Ces résultats } \\
\text { sont calculés à court terme. Il serait } \\
\text { intéressant qu'ils le soient aussi à moyen } \\
\text { terme. }\end{array}$ \\
\hline Les mesures & $\begin{array}{l}\% \text { de prêts honorés et \% de personnes } \\
\text { remises « en selle »: satisfaisants. } \\
\% \text { de personnes suivies : stable. }\end{array}$ \\
\hline L'écologie & $\begin{array}{l}\text { Système qui concerne l'ensemble des } \\
\text { parties prenantes de la banque } \\
\text { (sociétaires, employés, dirigeants) et qui } \\
\text { concerne le tissu économique territorial de } \\
\text { la banque. }\end{array}$ \\
\hline Les réseaux & $\begin{array}{l}\text { Le réseau des Points Passerelle est en } \\
\text { liaison avec d'autres réseaux } \\
\text { d'associations aidant de façon informelle } \\
\text { les personnes en difficulté. Il est en liaison } \\
\text { avec les réseaux des agences bancaires, } \\
\text { plus particulièrement le Crédit Agricole du } \\
\text { Nord Est. }\end{array}$ \\
\hline
\end{tabular}


Tableau 2 : Les résultats des actions menées au sein de la Caisse du Crédit Agricole du Nord Est.

\begin{tabular}{|l|c|}
\hline Succès complet & $51 \%$ \\
\hline Succès partiel & $15 \%$ \\
\hline Sans résultat significatif & $31 \%$ \\
\hline Échec & $3 \%$ \\
\hline
\end{tabular}

